\title{
Alexis de Tocqueville au Canada (du 24 août au 2 septembre
} 1831)

\section{Jean-Michel Leclercq}

Volume 22, numéro 3, décembre 1968

URI : https://id.erudit.org/iderudit/302795ar

DOI : https://doi.org/10.7202/302795ar

Aller au sommaire du numéro

Éditeur(s)

Institut d'histoire de l'Amérique française

ISSN

0035-2357 (imprimé)

1492-1383 (numérique)

Découvrir la revue

Citer cet article

Leclercq, J.-M. (1968). Alexis de Tocqueville au Canada (du 24 août au 2 septembre 1831). Revue d'histoire de l'Amérique française, 22(3), 353-364. https://doi.org/10.7202/302795ar d'utilisation que vous pouvez consulter en ligne.

https://apropos.erudit.org/fr/usagers/politique-dutilisation/ 


\section{ALEXIS DE TOCQUEVILLE AU CANADA * (du 24 août au 2 septembre 1831)}

Tocqueville passe peu de temps au Bas-Canada. Son séjour se limite à une quinzaine de jours, étape remarquablement mise à profit pour réunir une abondante documentation ${ }^{1}$. Il procède d'ailleurs à une étude systématique, commençant par la rédaction d'une première fiche à bord du navire qui relie Montréal à Québec: "CANADA, apparence extérieure". Puis ce sont les entrevues avec des personnalités canadiennes, des visites et, finalement, la rédaction des "Remarques générales". Les différentes étapes de l'enquête permettent de dresser le tableau synoptique suivant:

\begin{tabular}{|c|c|c|}
\hline 24 août 1831 & Montréal & $\begin{array}{l}\text { - visite au Père J. V. Quiblier, } \\
\text { supérieur du Séminaire } \\
\text { - entretien avec D. et C. Monde- } \\
\text { let, avocats au barreau de } \\
\text { Montréal }\end{array}$ \\
\hline 25 août 1831 & sur le Saint-Laurent & $\begin{array}{l}\text { - rédaction d'une première fiche: } \\
\text { CANADA: apparence exté- } \\
\text { rieure }\end{array}$ \\
\hline 26 août 1831 & Québec & $\begin{array}{l}\text { - entretien avec un négociant } \\
\text { anglais } \\
\text { - visite à l'un des tribunaux } \\
\text { civils }\end{array}$ \\
\hline 27 août 1831 & Québec & $\begin{array}{l}\text { - conversation avec M. John } \\
\text { Neilson, député à la Chambre } \\
\text { d'Assemblée } \\
\text { - visite au cabinet de lecture }\end{array}$ \\
\hline
\end{tabular}

* Extrait d'un mémoire pour le diplôme d'études supérieures de sciences politiques de la Faculté de Droit et de Sciences économiques de Lille, 1965, pages 79-94.

1 Alexis de Tocqueville, Oeuvres completes. Edition définitive publiée sous la direction de Jean-Paul Mayer. Tome I - De la démocratie en Amérique, vol. 1 et 2, 5e éd. (Paris, Gallimard, 1951); tome II - L'Ancien Régime et la Révolution, vol. 1 et 2, 8e éd. (Paris, Gallimard, 1952); tome V - Voyages en Sicile et aux Etats-Unis, vol. 1, 4e éd. (Paris, Gallimard, 1957). Les notes de voyage relatives au Canada sont réunies dans le cahier non alphabétique numéro 1 et à la rubrique "Canada" du cahier, pages $74-85,210-225$. A ces notes il convient d'ajouter l'essai: "Quinze jours dans le désert" qui fut écrit au cours du voyage canadien et qui se trouve également dans le tome V: $342 \mathrm{ss}$. 


$\begin{array}{ll}28 \text { août } 1831 & \text { Québec } \\ 29 \text { août } 1831 & \text { Québec } \\ 31 \text { août } 1831 & \text { Québec } \\ \text { 1er septembre 1831 } & \text { Québec } \\ 2 \text { septembre 1831 } & \text { Québec }\end{array}$

- excursion dans la campagne québécoise en compagnie de J. Neilson

- excursion à Beauport sans guide

- excursion à Saint-Thomas avec J. Neilson et D.-B. Viger

- rédaction des "Remarques générales"

- conversation avec J.-T. Taschereau, juge canadien

Les premiers jours sont utilisés à entrer en relations avec des interlocuteurs canadiens et à visiter les lieux publics, tandis que les derniers sont consacrés à des excursions hors de l'agglomération. Cela correspond bien aux deux mouvements de l'investigation entreprise: l'établissement d'une documentation et la vérification des renseignements obtenus.

Une méthode d'enquête utilisant le questionnaire. - Tocqueville pense que le meilleur moyen de sonder l'état d'esprit dominant d'une société donnée est de confier la parole à ses membres. Cependant la conversation ne va pas au gré des idées émises mais aborde plutôt successivement un certain nombre de sujets selon un plan sciemment établi. C'est un véritable sondage d'opinion que nous suivons au fil des entretiens rapportés. Tocqueville établit tour à tour, avec ses différents interlocuteurs, des rubriques qui forment autant de fiches dont l'exploitation lui permettra d'apprécier l'état de l'opinion publique au Bas-Canada. Pour pénétrer cette méthode des questionnaires, il convient d'étudier les interlocuteurs sélectionnés et les questions posées.

\section{A - Le choix des interlocuteurs}

Le Père Joseph-Vincent Quiblier est le premier personnage rencontré au Bas-Canada; c'est un ecclésiastique français, supérieur du Séminaire de Montréal. Sa qualité de Français établi depuis quelques années au Canada va permettre d'intéressantes comparaisons entre le clergé canadien et le clergé français. Tocqueville nous en laisse le portrait suivant: "Monsieur Quiblier nous a paru un ecclésiastique aimable et éclairé." ${ }^{2}$

2 Ibid., V: 77. 
Le même jour, se déroule la visite chez les frères Mondelet, "jeunes gens intelligents et de bon sens" ${ }^{3}$. Dominique et CharlesJ.-E. Mondelet appartiennent à une vieille famille de la bourgeoisie montréalaise. Ils sont tous deux avocats et, comme la plupart des Canadiens appartenant à la classe des professions libérales, ils s'intéressent vivement à la politique. La plupart des hommes politiques canadiens se recrutent, à cette époque, parmi les milieux du barreau, du notariat et du journalisme. Dominique Mondelet est député de Montréal à la Chambre d'Assemblée du Bas-Canada. Il appartient à la tendance modérée du parti d'opposition, c'est-à-dire qu'il milite pour l'application effective de l'Acte constitutionnel de 1791. Comme John Neilson, Dominique Mondelet estime que le gouvernement représentatif n'a pas encore véritablement existé au Canada, puisque l'oligarchie l'a confisqué à son profit, et il croit aux vertus de réformes progressives opérées dans le cadre du maintien de l'allégeance à la Couronne britannique. En 1832, le gouverneur Aylmer nommera Mondelet au Conseil exécutif et la tendance extrémiste de L.-J. Papineau demandera son expulsion de la Chambre. Battu aux élections de 1834, il se retirera de la scène politique et occupera ensuite un poste de magistrat.

Le troisième interlocuteur choisi est un marchand anglais de Québec dont l'anonymat est soigneusement protégé. Ce négociant appartient sans doute à la génération d'immigrants anglais qui vinrent s'installer après le traité de Paris et s'emparèrent rapidement du commerce et de la traite des fourrures. Ce choix semble guidé par l'intention de donner la parole à un adversaire caractéristique des Canadiens français. L'entretien, du reste, est laconique et ne porte que sur la question ethnique.

Les entretiens les plus féconds, Tocqueville les aura avec celui que l'on peut considérer comme son interlocuteur principal: John Neilson. Certaines erreurs s'étant glissées dans le portrait esquissé en préface à la conversation du 27 août, il convient de rappeler sommairement qui est John Neilson. Né en Écosse (et non au Canada) il est arrivé à Québec en 1790;

3 Ibid., V: 78. 
d'abord journaliste, rédacteur en chef de la Gazette de Québec, gagné à la cause canadienne, il milite dans le parti d'opposition. Neilson siège à l'Assemblée de 1818 à 1834. Après la crise de 1827 il est désigné pour présenter les revendications du parti populaire à Londres et s'y rend en compagnie de MM. D.-B. Viger et Augustin Cuvillier en 1828 (et non en 1825 comme le note Tocqueville). "Homme d'un esprit vif et original" ", Neilson fut, jusqu'aux années 1831-1832, l'ami et le partenaire politique de Louis-Joseph Papineau.

Bien que leurs entretiens ne soient pas rapportés, l'on sait que Tocqueville rencontra également Denis-Benjamin Viger et J.-T. Taschereau ${ }^{5}$, deux autres personnages politiques de cette époque.

\section{B - La nature des questions posées}

Tocqueville pose à chacun de ses interlocuteurs une série de questions correspondant aux cases d'une grille où sont répertoriés les différents centres d'intérêt. Ainsi peut-il s'informer de la situation ethnique, politique, sociale et religieuse du BasCanada. Tous les sujets ne sont pas forcément épuisés avec chaque interlocuteur, mais dans l'ensemble, chaque centre d'intérêt est abordé. La question raciale est envisagée au cours des quatre entrevues, tandis que les problèmes politiques sont réservés essentiellement à Neilson.

Tocqueville rapporte le résultat de ses entretiens sous la forme d'une conversation, mais il s'agit d'un dialogue artificiellement reconstitué. Les questions, en réalité, ont été beaucoup plus nombreuses et beaucoup plus précises. Déjà dans le journal du voyage en Sicile, l'auteur avait ainsi résumé les propos échangés entre deux personnages ${ }^{6}$.

Les différentes rubriques passées en revue lors des conversations permettent d'établir le questionnaire-type suivant:

4 Ibid., V: 80.

5 G. W. Pierson, Tocqueville and Beaumont in America (New York, 1938), index, 839.

6 Ibid., V : 50. 
a) situation ethnique -

1 - quelles sont les proportions respectives des deux populations ?

2 - quelle est la nature des rapports entre les deux communautés?

3 - peut-on envisager l'éventualité d'un soulèvement canadien-français?

b) situation politique -

1 - quels sont les rapports entre le Canada et l'Angleterre ?

2 - les Canadiens secoueront-ils le joug anglais ?

3 - dans l'affirmative, le risque n'est-il pas grand d'une absorption par les Etats-Unis ?

4 - quelle est l'influence de la presse (nombre de journaux, nombre d'abonnés, importance dans la vie politique du Bas-Canada)?

5 - quelles sont les modalités de la loi électorale ?

c) état social -

1 - régime de la propriété — quels sont les restes du régime féodal ?

2 - niveau de l'instruction - l'instruction progresse-t-elle ?

3 - état des mœurs - quel est le caractère du Canadien ? les mœurs sont-elles pures?

4 - mouvements de population - les Canadiens s'étendent-ils ?

5 - affaires indigènes - quelles sont les mœurs des Indiens ?

d) situation religieuse -

1 - quelle est la position du clergé vis-à-vis des habitants ?

2-quelle est l'attitude des habitants vis-à-vis du clergé ? (comportement de la presse, rôle de l'instruction).

En fait, chaque sujet évoqué est subdivisé en plusieurs questions secondaires. Si l'on analyse les réponses fournies par les quatre interlocuteurs, l'on remarque que les réponses sont faites dans les mêmes termes, ce qui permet de supposer qu'elles 
correspondent à des questions semblables et précises. Deux exemples sont caractéristiques. L'analyse de la conversation avec les frères Mondelet et avec Neilson portant sur la position du clergé permet de déduire que les questions étaient ainsi formulées:

- quelle est la position du clergé catholique ?

- le clergé catholique est-il politisé ?

- le clergé catholique canadien est-il différent dans son attitude politique du clergé européen et spécialement du clergé français?

- le clergé catholique fait-il corps avec la population dans ses revendications nationales?

- accuse-t-on le clergé catholique d'être démagogue ?

- le clergé catholique voit-il favorablement le développement de l'instruction?

La comparaison des réponses formulées par le Père Quiblier et John Neilson, à propos du régime de la propriété, laisse sousentendre les questions suivantes:

- à quels droits le seigneur peut-il prétendre ?

- quelles sont les sujétions imposées aux tenanciers?

- à combien s'élève la rente ?

- le seigneur jouit-il de droits honorifiques ?

- quelle est la position sociale du seigneur par rapport à l'habitant?

Tocqueville enregistre méthodiquement les réponses, mais note aussi l'attitude des interlocuteurs, leur humeur pendant l'entretien, c'est-à-dire tout ce qui peut indiquer, au-delà des termes de la réponse, un état d'esprit révélateur et significatif. Ainsi, lors de sa rencontre avec le négociant anglais, il observe dans une note: "En parlant des Canadiens, il se peignait sur la physionomie flegmatique de Mr.... un sentiment de haine et de mépris très visible." "

7 Ibid., V: 79. 
Ailleurs, il mentionne les précautions dont il entoure les questions relatives au problème racial au cours de la conversation avec Neilson.

La vérification et l'exploitation des résultats. - Quand Tocqueville a recueilli les divers renseignements par la méthode des questionnaires, il décide de vérifier la véracité des opinions émises. C'est le motif des visites des différents lieux publics et surtout de la chevauchée "sans guide" dans la campagne québécoise. "Nous nous sommes écartés dans les sentiers et nous avons causé avec tous les habitants que nous avons rencontrés, tâchant de faire porter la conversation sur des sujets graves." 8 Tout ce qui peut contribuer à apprécier l'état social du pays est minutieusement noté: niveau de vie, signes extérieurs de richesse, ornementation des logis et des églises, mais également tout ce qui permet de brosser un portrait national: reparties plaisantes, réflexions entendues au hasard des conversations.

Cette opération de vérification est indispensable. Tocqueville ne manque pas de relever à la fois ce que les faits démentent et ce qu'ils corroborent. Ainsi, au sujet de la dîme, il ne tarde pas à se rendre compte qu'elle n'est pas aussi facilement admise qu'on le lui avait laissé entendre. Neilson déclare: "Le Canadien est profondément religieux et paie la dîme sans répugnance." ${ }^{8}$

Le Père Quiblier affirme: "Elle est payée sans répugnance et sans peine." 10

Cependant l'avis des intéressés s'étant révélé fort différent au cours de la randonnée dans la campagne, Tocqueville écrit: “quoiqu'en dise Mr. Neilson ... nous avons cru remarquer aussi que le paysan ne voyait pas sans peine le droit que le clergé a de prélever la dîme". ${ }^{11}$

En revanche, l'explication fournie par les frères Mondelet de l'union étroite de la cause du clergé catholique et de la cause

8 Ibid., V: 214.

9 Ibid., V: 82.

10 Ibid., V: 77.

11 Ibid., (éd. dite Beaumont), VIII : 264. 
nationale française apparaît convaincante. L'on retrouve presque mot à mot les termes du questionnaire dans la lettre adressée à l'abbé Lesueur: "Loin d'être accusé d'être ici le partisan du pouvoir, les Anglais le traitent en démagogue. Le fait est qu'il est le premier à résister à l'oppression et le peuple voit en lui son plus constant appui. Aussi, les Canadiens sont-ils religieux par principe et par passion politique." 12

L'analyse des notes recueillies par l'auteur montre qu'il fait, à l'occasion de son voyage, une véritable enquête, méthodiquement dirigée, allant des choses aux hommes, des évidences sensibles aux idées personnelles, afin de dresser un tableau d'ensemble objectif de la situation du pays visité. Toute méthode, aussi rigoureuse soit-elle, implique pourtant de la part de celui qui l'utilise, une objectivité impartiale. Appliquée à l'étude de phénomènes humains en face desquels il est difficile de demeurer neutre, la démarche tocquevillienne va se révéler critiquable.

\section{Les faiblesses d'une enquête}

Une information insuffisante - Il a manqué à Tocqueville une documentation sérieuse sur les problèmes canadiens. Son itinéraire, nous l'avons vu, est conçu de telle façon qu'il ne connaîtra que la province française. Il voudra ignorer qu'une autre province a son sort lié à celle qu'il visite et que le mouvement populaire d'opposition a son homologue dans le HautCanada. Le questionnaire, bien que remarquablement élaboré, comporte cependant de graves lacunes dans le domaine de l'information économique. Au cours de l'entretien avec le négociant britannique, il n'est guère question que du problème ethnique. En 1831, le Canada connaît de nombreuses préoccupations économiques qui ne sont, à aucun moment, évoquées, ni dans les notes, ni au cours des conversations. A peine Tocqueville observe-t-il que toutes les enseignes sont rédigées en langue anglaise et que les Anglais détiennent toute la richesse, mais aucune étude sérieuse n'est entreprise en ce domaine. Le problème politique n'est abordé que dans le cadre des rivalités ethniques.

12 Ibid., VII : 57. 
Il n'est nulle part question de l'Acte de 1791 et des institutions constitutionnelles établies par cette loi. La réforme des conseils, l'indépendance des juges, le revenu des biens des Jésuites, l'importante question des subsides qui se trouvent au centre de la vie politique canadienne pendant toute cette période, sont passés sous silence. La rubrique sociale, plus abondante, comporte aussi de graves lacunes. Tocqueville renouvelle les questions ayant trait aux vestiges du régime féodal, mais méconnaît les réserves de terrains auxquelles le gouvernement procède au profit de la Couronne, de même que le débat qui oppose les partisans du régime anglais de propriété à leurs adversaires français dans les Cantons de l'Est. A propos de l'instruction, nous apprenons qu'elle se répand, mais nous continuons d'ignorer son statut. Nulle mention qu'en 1831 le budget de l'instruction publique favorise essentiellement les écoles de l'Institution Royale anglicisante. ${ }^{13}$

Les relations d'un aristocrate - L'on peut reprocher à l'auteur de n'avoir pas su recueillir l'avis de gens capables de le renseigner valablement. De là cette documentation incomplète et lacuneuse.

Tocqueville voyage en homme du monde. Les gens qu'il rencontre et qui déterminent son opinion ne sont pas les plus caractéristiques, mais simplement ceux vers qui l'envoient des lettres de recommandation. L'ouvrage de Monsieur G. W. Pierson permet de reconstituer le cercle des relations de l'auteur pendant son séjour au Bas-Canada.

Le révérend John Power de New York lui avait remis une lettre à l'adresse du Père Quiblier, supérieur du Séminaire de Montréal, ce qui lui vaut de rencontrer son premier interlocuteur.

Un "légiste très distingué", membre du Congrès, John Spencer, de Canandaigua, lui avait remis un mot d'introduction auprès des frères Mondelet. Ce sont ces derniers qui le recommandent

13 Mason Wade, Les Canadiens fransais de 1760 à nos jours. Traduit de l'anglais par Adrien Vienne (Ottawa, Cercle du livre français, 1953), I: 161 . 
à John Neilson en lui remettant, le 24 août 1831, le billet suivant: "Je ne peux rien faire de mieux que d'envoyer ces deux Messieurs qui voyagent afin de connaître le pays et ses habitants à un homme comme vous qui sera certainement capable de leur fournir d'utiles et intéressants renseignements." 14

John Neilson fera les honneurs de Québec aux deux Français et leur présentera Denis-Benjamin Viger et Jean-Thomas Taschereau. Hasard des rencontres de voyage qui permet à Tocqueville de faire la connaissance d'amis canadiens. Sa qualité de fonctionnaire français en mission, son rang social auraient pu lui ouvrir toutes les portes de la société québécoise, y compris celle du gouverneur Aylmer. Cependant Tocqueville ne cherche pas à s'en prévaloir et préfère la méthode empirique d'un "honnête homme" en voyage. Une objectivité rigoureuse eût exigé qu'il consultât chacun des deux partis en présence, c'est-à-dire, d'une part, un membre du gouvernement, le gouverneur ou l'un de ses conseillers et, d'autre part, celui qui est le chef de l'opposition: Louis-Joseph Papineau. Ceux qu'il rencontre ne sont, au contraire, guère représentatifs: pour le clergé, un prêtre français, et pour le personnel politique, un député qui sera, l'année suivante, exclu de son parti et déclaré "traître à sa race" (D. Mondelet) et un chef politique dont l'influence diminue graduellement et qui se retirera trois ans plus tard (J. Neilson).

Aux États-Unis l'on a reproché à Tocqueville d'avoir trop subi l'influence des milieux de la Banque et des hommes politiques de Nouvelle-Angleterre et de s'être conformé un peu hâtivement à leurs opinions. Sans doute peut-on lui faire un reproche identique au Canada. Le fait de n'être pas entré en relations avec un personnage comme Louis-Joseph Papineau est, à ce sujet, suffisamment révélateur.

Un parti pris français évident - Tocqueville éprouve une profonde sympathie pour le peuple canadien. Une communauté de sentiments ne tarde pas à s'établir entre ces gens courtois, honnêtes et simples, restés foncièrement Français de cœur, et cet

${ }^{14} \mathrm{G}$. W. Pierson, Tocqueville and Beaumont in America (New York, Oxford University Press, 1938), 806. 
aristocrate dont la ferveur patriotique bouillonne d'autant plus vivement qu'il est éloigné du sol natal. C'est la France aux fleurs de lys qu'il retrouve au Bas-Canada: "Nous avons retrouvé là, surtout dans les villages éloignés des villes, les anciennes habitudes, les anciennes mœurs françaises. Autour d'une église surmontée du coq et de la croix fleurdelisée..." 15 Et cependant cette "vieille France" a évolué vers la démocratie sans déchirement. Comment dès lors ne pas partager les aspirations d'un peuple qui concilie si harmonieusement liberté et démocratie, religion et politique et lui témoigne tant de cordialité. Le Canada est une découverte qui enthousiasme Tocqueville, mais le révolte dès qu'il prend conscience de la situation d'infériorité dans laquelle les Anglais maintiennent les Canadiens.

Les frères Mondelet ont révélé aux deux voyageurs l'acuité du problème de la survivance française et désormais le conflit ethnique est au centre de leurs préoccupations. L'entretien avec le négociant anglais, nous l'avons dit, est entièrement consacré à la question raciale. Tocqueville est profondément troublé dans sa conscience de magistrat par le spectacle burlesque du tribunal, et ce n'est point la lecture des colonnes du Canadien qui peut le détourner de sa première impression. "Le plus beau rejeton de la famille européenne dans le Nouveau-Monde" ${ }^{16}$ est honteusement humilié par l'Anglais qu'il appelle bientôt: "le peuple conquérant", puis "l'ennemi". A partir de ce moment, l'examen serein de la réalité sociale est bouleversé par la tentation de l'action politique. Son enquête consiste alors à se demander si les Canadiens sont ou non capables de secouer le joug anglais et, dans l'affirmative, par quels moyens.

Les classes éclairées semblent s'accommoder trop facilement de la domination anglaise et Tocqueville décide d'en savoir plus long sur les sentiments qui animent vraiment la masse des habitants. Telle semble être la raison de ces promenades "sans guide" dans la campagne québécoise, prétextes à des questions VII : 56.

${ }^{16}$ Alexis de Tocqueville, Oeuvres complètcs (Edition Beaumont), 16 Ibid., VII : 87. 
orientées sur le conflit racial: "On voit que tout ce qu'on dit à ce sujet remue leurs passions, mais ils ne voient pas clairement le remède." 17

Ce remède, Tocqueville semble y aspirer plus qu'il ne convient à un observateur objectif.

Amené à utiliser sa méthode d'observation sociologique à l'étude d'une situation où il lui est malaisé de demeurer neutre, Tocqueville en modifie à tel point l'application qu'elle ne fournit pas tous les résultats escomptés.

Réflexion sur le Canada de 1831

Dans une lettre adressée à son frère, le 26 novembre 1831, Tocqueville résume l'impression d'ensemble que lui laisse son bref séjour au Canada:

"Je viens de voir dans le Bas-Canada un million de Français, braves, intelligents, faits pour former un jour une grande nation française en Amérique, qui vivent en quelque sorte en étrangers dans leur pays. Le peuple conquérant tient le commerce, les emplois, la richesse, le pouvoir. Il forme les hautes classes et domine la société entière. Le peuple, conquis, partout où il n'a pas l'immense supériorité numérique perd peu à peu ses mœurs, sa langue, son caractère national." 18

5400, avenue Decelles, app. 15

JEAN-MICHEL LECLERCQ

Montréal 250 $\mathrm{V}: 214$. VII : 88 .

17 Alexis de Tocqueville, Oeuvres complètes (éd. J. P. Mayer, op. cit.), 18 Alexis de Tocqueville, Oeuvres complètes (Edition Beaumont), 